Published in final edited form as:

J Eur Acad Dermatol Venereol. 2020 January ; 34(1): 74-81. doi:10.1111/jdv.15819.

\title{
Reflectance confocal microscopy and dermoscopy aid in evaluating repigmentation within or adjacent to lentigo maligna melanoma surgical scars
}

\author{
C. Navarrete-Dechent ${ }^{1,2}$, M. Cordova ${ }^{1}$, K. Liopyris ${ }^{1}$, A. Rishpon ${ }^{1,3}$, S. Aleissa ${ }^{1}$, A.M.

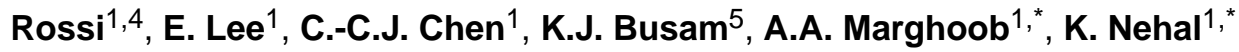 \\ ${ }^{1}$ Dermatology Service, Department of Medicine, Memorial Sloan Kettering Cancer Center, New \\ York, NY, USA \\ ${ }^{2}$ Department of Dermatology, Escuela de Medicina, Pontificia Universidad Catolica de Chile, \\ Santiago, Chile \\ ${ }^{3}$ Department of Dermatology, Tel Aviv Sourasky Medical Center, Tel Aviv, Israel \\ ${ }^{4}$ Weill Cornell Medical College, New York, New York, USA \\ ${ }^{5}$ Department of Pathology, Memorial Sloan Kettering Cancer Center, New York, NY, USA
}

\begin{abstract}
Background: Determining whether repigmentation within or adjacent to lentigo maligna or lentigo maligna melanoma (LM/LMM) scars represents recurrence of melanoma is challenging. The use of reflectance confocal microscopy (RCM) and dermoscopy may aid in differentiating true melanoma recurrence from other causes of repigmentation.
\end{abstract}

Objectives: To describe the characteristics of repigmentation within or adjacent to LM/LMM scars observable on RCM and dermoscopy.

Methods: We retrospectively analysed patients who presented with new pigmentation within or adjacent to scars from surgically treated LM/LMM between January 2014 and December 2018. Clinical and demographic characteristics and time to recurrence were recorded. RCM was used to evaluate areas of pigmentation before biopsy. If available, dermoscopic images were evaluated.

Results: In total, 30 confocal studies in 29 patients were included in the study cohort. Twentyone patients had biopsy-confirmed recurrent LM/LMM; the remainder had pigmented actinic keratosis $(\mathrm{n}=4)$ or hyperpigmentation/solar lentigo $(\mathrm{n}=5)$. RCM had sensitivity of $95.24 \%(95 \%$

Correspondence to: Kishwer S. Nehal, MD, Dermatology Service, Department of Medicine, Memorial Sloan Kettering Cancer Center, 16 E. 60th Street, New York, NY 10022, nehalk@mskcc.org, Phone: +1646-888-6022.

A.A.M. and K.N. share senior authorship.

Disclosures:

A. A. Marghoob: Received honorarium from 3GEN for dermoscopy lectures, royalties from publishing companies for books and book chapters, dermoscopy equipment for testing and feedback, and payment from the American Dermoscopy Meeting for organizing and lecturing at the annual meeting.

A. Rossi: Dr. Rossi has no relevant conflicts of interest related to this manuscript but has received grant funding from The Skin Cancer Foundation and the A.Ward Ford Memorial Grant for research related to this work. He also served on advisory board, as a consultant, or given educational presentations: for Allergan, Inc; Galderma Inc; Evolus Inc; Elekta; Biofrontera, Quantia; Merz Inc; Dynamed;

Skinuvia, Perf-Action, and LAM therapeutics. 
CI, 76.18\%-99.88\%), specificity of 77.7\% (95\% CI, 39.99\%-97.19\%), positive predictive value of $90.91 \%$ (95\% CI, 74.58\%-97.15\%), and negative predictive value of $87.5 \%$ (95\% CI,

50.04\%-98.0\%). The most common dermoscopic feature observed among patients with recurrent LM/LMM was focal homogeneous or structureless areas of light-brown pigmentation (92.8\% vs $37.5 \%$ in patients with other diagnoses; $\mathrm{P}=0.009)$. LM-specific dermoscopic criteria were present in only $28.5 \%$ of patients with recurrent LM/LMM.

Conclusions: RCM and dermoscopy are valuable tools for the comprehensive evaluation of repigmentation within or adjacent to LM scars.

\section{Keywords}

lentigo maligna; melanoma; reflectance confocal microscopy; recurrence; dermoscopy; dermatoscopy; pigmentation; repigmentation

\section{Introduction}

Lentigo maligna and lentigo maligna melanoma (LM/LMM) are the most common melanoma subtypes on the head and neck. ${ }^{1} \mathrm{LM} / \mathrm{LMM}$ often harbour subclinical extensions, which contribute at least in part, to the higher rates of local recurrence, compared with other melanoma subtypes. ${ }^{1-5}$ As LM/LMM develops on chronically sun-exposed areas, hyperpigmentation and formation of lentigines around melanoma scars is not uncommon. This new pigmentation within or adjacent to an LM/LMM surgical scar may pose a diagnostic challenge in differentiating recurrent melanoma from other diagnoses. ${ }^{3-6}$ Although biopsy remains the gold standard for diagnosing recurrent LM/LMM, limitations of biopsy include additional scarring, cost, time, and potential sampling error leading to false-negative diagnosis.

Reflectance confocal microscopy (RCM) and dermoscopy are noninvasive imaging modalities that have high accuracy for diagnosis of primary LM/LMM. ${ }^{7,8}$ The use of RCM and dermoscopy for the diagnosis of recurrent $\mathrm{LM} / \mathrm{LMM}$ is now being explored. ${ }^{9-11}$ Cinotti et al. showed that RCM had a higher sensitivity than dermoscopy for recurrent LM when compared to benign pigmented facial macules. ${ }^{11}$ Herein, we evaluated the role of RCM and dermoscopy in identifying LM/LMM recurrence in consecutive patients with recurrent pigmentation within or adjacent to a scar resulting from the surgical removal of LM/LMM.

\section{Patients and methods}

The institutional review board at Memorial Sloan Kettering approval this study (\#99-099). All patients gave written informed consent. We reviewed our database from January 2014 to December 2018 for patients that presented with a clinical diagnosis of "possible recurrent LM" based on new areas of pigmentation within or adjacent to a surgical scar from a previous LM/LMM excision. Patients with a diagnosis of LM/LMM who were initially treated with nonsurgical techniques, such as imiquimod or radiation, were excluded from this study as they have been analysed previously. ${ }^{10}$ Initial surgical treatment was performed either at our cancer center or elsewhere. Demographics, clinical information, estimated size of repigmentation (diameter), images (clinical, dermoscopic, and RCM), and pathology 
reports were retrieved from patient medical charts. Clinical and dermoscopic images were acquired using the same digital camera (Veos DS3; Canfield Scientific, NJ, USA).

\section{RCM evaluation:}

RCM images were obtained prior to biopsy using a handheld RCM device (VivaScope 3000, Caliber ID, Rochester, NY) by 3 investigators (C.N-D, M.C., and K.L). Single images, stacks (from corneal layer to superficial dermis), and videos were obtained from the areas of pigmentation with the aid of paper rings, as previously described. ${ }^{12}$ The number of images/ stacks/videos obtained varied case-by-case with the intent to evaluate the complete area of repigmentation. The pigmented areas were evaluated for the presence or absence of RCMspecific melanoma criteria, including bright large nucleated (dendritic or roundish) cells at the dermoepidermal junction (DEJ), suprabasal epidermis or superficial dermis, with or without perifollicular localization. ${ }^{13,14}$ RCM images were deemed "RCM-positive" or "RCM-negative" based on the Guitera et al criteria. ${ }^{7}$ RCM images were evaluated by 3 investigators (C.N.-D., M.C., and K.L.) who were blinded to the final histopathologic diagnosis.

\section{Dermoscopic examination}

Dermoscopic images were evaluated for LM/LMM-specific criteria, including circles (regular or irregular), circles within circles, annular-granular structures, angulated lines, and obliteration of hair follicles. ${ }^{8}$ Images were also analysed for the dermoscopic criteria described in the latest consensus by Kittler et al. ${ }^{15}$ Evaluation was performed for consensus by 2 investigators (C.N.-D. and A.A.M.) who were blinded to the final histopathologic diagnosis.

\section{Histopathologic analysis}

Biopsy specimens were obtained under RCM guidance from areas deemed to be concerning for melanoma on the basis of RCM criteria. ${ }^{7}$ All biopsy specimens were reviewed by an expert dermatopathologist (K.J.B.). Cases were labelled "melanoma" or "other diagnosis." Results from RCM and dermoscopy were correlated with the final histopathologic diagnosis to evaluate the correlation between histopathologic examination and RCM or dermoscopy.

\section{Statistical analysis}

Data were analysed using SPSS 23.0 (SPSS, Armonk, NY, USA). Unless otherwise noted, all values are expressed as mean \pm standard deviation. Fisher's square test was used for categorical variables. Odds ratios (ORs) were calculated. Independent-samples student's $t$ test was performed for comparisons of continuous variables. A 2-tailed $\mathrm{P}<0.05$ was considered to indicate statistical significance. Sensitivity, specificity, positive predictive value, and negative predictive value of the RCM findings and final histopathological diagnosis was calculated.

\section{Results}

We performed 30 RCM examinations on 29 patients who presented with new areas of pigmentation within or adjacent to scars from prior excision of LM/LMM. One patient 
presented with a second repigmentation 2 years apart (Supplementary Table 1). Twenty-nine out of thirty lesions (96.6\%) were located on the head and neck area: One patient had a lesion on the lower leg. Age at initial diagnosis of melanoma was $64.5 \pm 11.2$ years (46-86 years). The male:female ratio was $1: 1$. Mean repigmentation size was $1.75 \mathrm{~cm} \pm 1.2$; range $0.5-5.0 \mathrm{~cm})$. Eight melanomas $(26.7 \%)$ were invasive. The median Breslow thickness among invasive melanomas was $0.25 \pm 0.35 \mathrm{~mm}(0.15-1.2 \mathrm{~mm})$. Demographic data are listed in Supplementary Table 1.

\section{Clinical features and histopathologic diagnosis}

Details of the cohort, by histopathologic diagnosis, are listed in Table 1. All patients presented with pigmented macules within or adjacent to the original scar. Definitive diagnosis of recurrent LM/LMM in areas of pigmentation was histologically confirmed for $21(70.0 \%)$ of the 30 cases. The remaining 9 patients (30.0\%) had a non-LM diagnosis: 4 with pigmented actinic keratosis (pAK) and 5 with a background of hyperpigmentation/solar lentigo. Age at recurrence of pigmentation was $71.1 \pm 12.0$ years among patients with $\mathrm{LM} / \mathrm{LMM}$ and $70.7 \pm 7.0$ years among those with other diagnoses $(\mathrm{P}=0.92)$. Time to recurrence of pigmentation was $68.1 \pm 43.4$ months (13-180 months) among LM/LMM patients and $52.4 \pm 39.1$ months among those with other diagnoses $(\mathrm{P}=0.36)$. There was no difference between size and diagnosis $(\mathrm{p}=0.45)$.

\section{RCM features:}

RCM had an overall diagnostic accuracy of 90.0\% (95\% CI, 73.47\%-97.89\%) and correctly identified true recurrent LM/LMM from other causes in 20 (95.2\%) of 21 cases (Table 1; Figs 1-4). The OR for diagnosis of recurrent LM/LMM with RCM was 70.0 (95\% CI, 5.46-896.59) with an area under the curve (AUC) of 0.865 . RCM had sensitivity of $95.24 \%$ (95\% CI, 76.18\%-99.88\%), specificity of 77.7\% (95\% CI, 39.99\%-97.19\%), positive predictive value of $90.91 \%$ (95\% CI, 74.58\%-97.15\%), and negative predictive value of $87.5 \%$ (95\% CI, 50.04\%-98.0\%). On RCM, 20 (95.2\%) of the 21 patients with LM/LMM had bright large nucleated cells at the DEJ/suprabasal epidermis (Figures 1 and 2). Of the 9 examinations with other diagnoses, 2 (22.2\%; both with pAK) had bright large nucleated cells at the DEJ ( $\mathrm{P}<0.001$; Figures 3 and 4$)$. Nucleated cells with folliculotropism were observed in $18(85.7 \%)$ of the 21 cases of LM/LMM and in 1 of the 9 cases of other diagnosis $(11.1 \%)(\mathrm{P}<0.001)$. Of the 9 patients with non-LM/LMM diagnoses, $8(88.8 \%)$ had a cobblestone pattern $(\mathrm{P}=0.014)$ and $4(44.4 \%)$ had an atypical honeycomb pattern $(\mathrm{P}=1.0)$. Bright nucleated cells above the suprabasal layer were seen more commonly in larger lesions ( $2.1 \mathrm{~cm}$ vs $0.9 \mathrm{~cm} ; \mathrm{p}=0.01$ ). No other RCM feature was associated with size of recurrent pigmentation in the recurrent melanoma group (data not shown).

\section{Dermoscopic features:}

Dermoscopic images were available for $22(69.2 \%)$ of the 26 cases (Table 1). The most common dermoscopic feature in patients with recurrent LM/LMM was 'focal homogeneous or structureless areas of light-brown pigmentation' (92.8\% vs $37.5 \%$ in patients with other diagnoses; $\mathrm{P}=0.009)$. LM-specific dermoscopic criteria were present in only $28.6 \%(4 / 14)$ of patients with recurrent LM/LMM (Table 1). Patients with non-LM causes of pigmentation commonly had fingerprinting and a pseudonetwork identified on dermoscopy (37.5\% vs 
$14.2 \%$ and $25 \%$ vs $0 \%$ in patients with $\mathrm{LM} / \mathrm{LMM}$, respectively; these results were not statistically significant $\mathrm{P}=0.309$ and 0.121 ). No dermoscopic feature was associated with size of recurrent pigmentation in the recurrent melanoma group (data not shown).

\section{Discussion:}

Evaluation of new areas of pigmentation within or adjacent to surgically excised LM/LMM scars can be challenging. The differential diagnosis includes lentigines, postinflammatory hyperpigmentation, pAK, and melanoma recurrence. Poikilodermatous changes within the scar often contribute to the diagnostic confusion. Until recently, the decision to biopsy an area of repigmentation within or adjacent to an LM/LMM scar has commonly been based on clinical suspicion, with or without the aid of dermoscopy. Dermoscopy was of limited benefit in our cohort. In two-thirds of cases, only a homogeneous or structureless lightbrown pigment, without any melanoma-specific structures, was identifiable under dermoscopy. In contrast, positive findings on RCM were strongly associated (OR, 70.0) with the final histopathologic diagnosis, confirming that RCM may play a role in differentiating recurrent LM/LMM from other causes of pigmentation. Scar tissue did not appear to hinder the detection of recurrent LM/LMM by RCM: The sensitivity and specificity for detecting recurrent LM/LMM associated with scars in this cohort was similar to previous studies assessing LM/LMM with no associated scars. ${ }^{7,11,16}$

Recurrence appears to be a late phenomenon in patients with LM/LMM, as it can occur 5 to 10 years after initial surgery. $3,6,17$ We observed a mean time to recurrence of repigmentation of 5.6 years, with 1 patient experiencing a melanoma recurrence 16 years after initial treatment. ${ }^{18}$ The mean age at recurrence was 71.1 years; these patients have a long-life expectancy, and the management of recurrent melanoma is complex. RCM and dermoscopy can be used as complementary tools for surveillance in cases where recurrence is suspected.

Previous studies evaluating RCM features in patients with recurrent LM after surgery have included 1 case report ${ }^{9}$ and 1 small case-series. ${ }^{17}$ Guitera et al. ${ }^{14}$ demonstrated that RCM is a useful tool to detect treatment failure after nonsurgical therapies (e.g., radiotherapy, imiquimod), with sensitivity of $94 \%$ and specificity of $100 \%$. Our results are similar to those of a prospective study evaluating RCM for the diagnosis of flat facial lesions that included LM/LMM, which found an overall sensitivity of $95 \%$ and specificity of $82 \% .{ }^{16}$ Additionally, a study by Cinotti et al included 17 recurrent LM/LMM in a series of 223 facial lesions. They compared recurrent LM to any pigmented facial macules arising on normal skin as controls and found a sensitivity of $75 \%$ and a specificity of $46 \% .{ }^{10}$ Our comparison group, however, included repigmentation arising on/within scars and not pigmented facial macules arising on normal skin. Overall, these studies highlight that evaluation of recurrent lesions arising within scars may be complex.

Additionally, RCM can be used to select the optimal biopsy site within the scar. Selecting the optimal biopsy site in large areas of pigmentation may be challenging and can lead to false-negative results due to sampling error. Many of our patients presented with complex prior reconstructions (e.g., flaps, grafts) at the surgery site and/or large lesions that were commonly located on highly cosmetic and functional areas-evaluation of these sites may 
be difficult by the naked eye alone. RCM can be used to scout large lesional areas and select the site with the most concerning features for biopsy. This was highlighted by Mataca et al., they found that RCM could be used to select biopsy sites containing more LM histopathologic criteria in comparison to those identified by the naked eye. ${ }^{19}$

We identified 'homogeneous or structureless light-brown areas of pigmentation' as an important dermoscopic feature for the diagnosis of recurrent LM/LMM. Interestingly, more than two-thirds of cases in our cohort lacked classical dermoscopic criteria for LM/LMM. ${ }^{8}$ We hypothesize that this homogeneous or structureless light-brown pigmentation occurs because of a lentiginous proliferation of pigmented melanocytes in an epidermis with attenuated rete ridges and few adnexal openings, possibly due to previous inflammation and scarring. Most of the classic LM/LMM-specific features are related to the hair follicles (e.g., asymmetrical follicular openings, circles within circles, angulated lines, rhomboid structures); however, in patients with scar tissue (which is nearly devoid of hair follicles) from previous treatment of LM/LMM, melanoma-specific features would not be apparent on dermoscopy. It is important to recognize diffuse structureless pigmentation as a common feature seen under dermoscopy, and not to expect the classic dermoscopic features of facial melanoma to avoid missing a recurrent LM/LMM. In a previous study of 5 cases of recurrent LM analysed by dermoscopy, "diffuse pigmentation" was identified in 4 (80\%) cases, and only 1 had melanoma-specific criteria. ${ }^{17}$ Cinotti et al. reported a sensitivity of $61 \%$ and specificity of $92 \%$ for detection of LM by dermoscopy; however, the sensitivity and specificity of dermoscopy decreased to $55 \%$ and $56 \%$ for recurrent LM, respectively. ${ }^{10}$ This underscores the limitations and lack of specificity of dermoscopy for detecting recurrent LM/LMM. Diffuse homogeneous light-brown pigmentation was not exclusively seen in melanomas, as it was associated with other lesions arising in a previous scar. This pigmentation was seen in 3 of our cases with other causes of pigmentation (Figure 4).

\section{False negatives and false positives under RCM:}

RCM had sensitivity of $\sim 95 \%$ in our study. One case of recurrent melanoma was misclassified as non-LM (false-negative) by RCM: a 51-year-old woman who presented to our clinic with a recurrent pigmentation appearing on a previously treated 0.3-mm LMM on the neck. The recurrence occurred 54 months after the initial surgery. RCM examination revealed only diffuse inflammatory cells as bright dots and plump cells. On further review, one or two isolated large dendritic cells were observed at the DEJ in the periphery; however, no round or dendritic bright large cells with perifollicular distribution were seen.

Histopathologic analysis revealed an early recurrent LM. Dermoscopy showed a homogeneous brown pigmented area that, on the basis of our results, should prompt a biopsy. In such cases, RCM and dermoscopy should be used as complementary tools.

In our study, the most common cause of misclassification of cases as false-positives by RCM was pAK: 2 cases of pAK fulfilled the criteria for $\mathrm{LM}$ on RCM described by Guitera et al. (Figure 4$){ }^{7} \mathrm{pAK}$ is considered by many to be one of the most challenging differentials of LM/LMM by both dermoscopy ${ }^{20}$ and confocal microscopy. ${ }^{21,22}$ pAKs have been previously shown to display large dendritic cells under RCM in a similar manner to LM/LMM, with a specificity of only $53 \%$ in nonsurgically treated sites, making their differentiation 
challenging. ${ }^{22,23}$ Accordingly, when the RCM differential includes these two diagnoses in a previously treated LM/LMM, we suggest performing a biopsy to avoid missing a melanoma. In our study, only 1 case of pAK had folliculotropism. As suggested by Persechino et al., presence of folliculotropism may help to distinguish the two diagnoses. ${ }^{22}$ In the same study, atypical honeycomb pattern was not a reliable criterion for differentiation between LM/LMM and AK. In our series, atypical honeycomb was seen similarly in both groups $(\sim 40 \% ; \mathrm{p}=1.0)$. The diagnostic challenge in differentiating $\mathrm{pAK}$ and solar lentigo from a true melanoma recurrence is not limited to RCM, as differentiation on histopathologic analysis can also be challenging. ${ }^{22,24}$ It could be that RCM is able to detect subtle repigmentation earlier, thus adding to the number of false-positives.

\section{Limitations}

This was a retrospective study from a tertiary cancer center: Our population tends to be biased toward more-complex cases, and hence, our cohort included a higher proportion of recurrent melanomas than benign cases of scar repigmentation. It is possible that in a less complex setting, the proportion of benign causes of pigmentation may be higher. This is important since pAK may be confused with LM clinically, dermoscopically, and under RCM, and further studies on larger numbers of cases are required to better understand the differences between pAK and LM in association with scars. ${ }^{23,25}$ In addition, we had a relatively small sample size; however, to the best of our knowledge, this is the largest study to evaluate the use of RCM for recurrent melanoma after surgery to date. This is a rare and complicated scenario; as such, our results could help improve management of these complex cases. Finally, there was no correlation with the level of follicular involvement (i.e. infundibulum, isthmus); future studies should consider stratifying the level of involvement to correlate different RCM patterns given the frequent follicular involvement seen in LM. ${ }^{26}$

\section{Conclusions}

Recurrent LM/LMM often present with homogeneous or structureless light-brown pigmentation devoid of melanoma-specific structures on dermoscopy. RCM can help identify true recurrent LM/LMM on the basis of the presence of bright large nucleated cells at the DEJ/suprabasal epidermis with perifollicular localization. RCM can also assist in selecting the optimal area to biopsy with the highest likelihood of containing histopathologic features of LM. When faced with evaluating these diagnostically challenging cases, dermoscopy and RCM should be used as complementary tools.

\section{Supplementary Material}

Refer to Web version on PubMed Central for supplementary material.

\section{Acknowledgments}

David B. Sewell, of the Memorial Sloan Kettering Department of Surgery, provided editorial assistance.

Funding source:This research was funded, in part, by National Institutes of Health/National Cancer Institute Cancer Center Support Grant P30 CA008748. The funder played no role in any aspect of the study. 


\section{References}

1. Fosko SW, Navarrete-Dechent CP, Nehal KS. Lentigo Maligna-Challenges, Observations, Imiquimod, Confocal Microscopy, and Personalized Treatment. JAMA Dermatol. 2018.

2. Osborne JE, Hutchinson PE. A follow-up study to investigate the efficacy of initial treatment of lentigo maligna with surgical excision. Br J Plast Surg. 2002;55(8):611-615. [PubMed: 12550112]

3. Connolly KL, Nijhawan RI, Dusza SW, Busam KJ, Nehal KS. Time to local recurrence of lentigo maligna: Implications for future studies. J Am Acad Dermatol. 2016;74(6):1247-1248. [PubMed: 27185424]

4. Hazan C, Dusza SW, Delgado R, Busam KJ, Halpern AC, Nehal KS. Staged excision for lentigo maligna and lentigo maligna melanoma: A retrospective analysis of 117 cases. J Am Acad Dermatol. 2008;58(1):142-148. [PubMed: 18029055]

5. Kunishige JH, Brodland DG, Zitelli JA. Surgical margins for melanoma in situ. J Am Acad Dermatol. 2012;66(3):438-444. [PubMed: 22196979]

6. Connolly KL, Hibler BP, Lee EH, Rossi AM, Busam KJ, Nehal KS. Locally Recurrent Lentigo Maligna and Lentigo Maligna Melanoma: Characteristics and Time to Recurrence After Surgery. Dermatol Surg. 2017;43(6):792-797. [PubMed: 28296789]

7. Guitera P, Pellacani G, Crotty KA, et al. The impact of in vivo reflectance confocal microscopy on the diagnostic accuracy of lentigo maligna and equivocal pigmented and nonpigmented macules of the face. J Invest Dermatol. 2010;130(8):2080-2091. [PubMed: 20393481]

8. Schiffner R, Schiffner-Rohe J, Vogt T, et al. Improvement of early recognition of lentigo maligna using dermatoscopy. J Am Acad Dermatol. 2000;42(1 Pt 1):25-32.

9. Longo C, Moscarella E, Pepe P, et al. Confocal microscopy of recurrent naevi and recurrent melanomas: a retrospective morphological study. Br J Dermatol. 2011;165(1):61-68. [PubMed: 21410674]

10. Guitera P, Haydu LE, Menzies SW, et al. Surveillance for treatment failure of lentigo maligna with dermoscopy and in vivo confocal microscopy: new descriptors. Br J Dermatol. 2014;170(6):13051312. [PubMed: 24641247]

11. Cinotti E, Labeille B, Debarbieux S, et al. Dermoscopy vs. reflectance confocal microscopy for the diagnosis of lentigo maligna. J Eur Acad Dermatol Venereol. 2018.

12. Marino ML, Rogers T, Sierra Gil H, Rajadhyaksha M, Cordova MA, Marghoob AA. Improving lesion localization when imaging with handheld reflectance confocal microscope. Skin Res Technol. 2016;22(4):519-520. [PubMed: 26792361]

13. Segura S, Puig S, Carrera C, Palou J, Malvehy J. Development of a two-step method for the diagnosis of melanoma by reflectance confocal microscopy. J Am Acad Dermatol. 2009;61(2):216-229. [PubMed: 19406506]

14. Guitera P, Menzies SW, Longo C, Cesinaro AM, Scolyer RA, Pellacani G. In vivo confocal microscopy for diagnosis of melanoma and basal cell carcinoma using a two-step method: analysis of 710 consecutive clinically equivocal cases. J Invest Dermatol. 2012;132(10):2386-2394. [PubMed: 22718115]

15. Kittler H, Marghoob AA, Argenziano G, et al. Standardization of terminology in dermoscopy/ dermatoscopy: Results of the third consensus conference of the International Society of Dermoscopy. J Am Acad Dermatol. 2016;74(6):1093-1106. [PubMed: 26896294]

16. Wurm E, Pellacani G, Longo C, et al. The value of reflectance confocal microscopy in diagnosis of flat pigmented facial lesions: a prospective study. J Eur Acad Dermatol Venereol. 2017;31(8):1349-1354. [PubMed: 28214381]

17. Erfan N, Kang HY, Cardot-Leccia N, et al. Reflectance confocal microscopy for recurrent lentigo maligna. Dermatol Surg. 2011;37(10):1519-1524. [PubMed: 21790847]

18. Donigan JM, Hyde MA, Goldgar DE, Hadley ML, Bowling M, Bowen GM. Rate of Recurrence of Lentigo Maligna Treated With Off-Label Neoadjuvant Topical Imiquimod, 5\%, Cream Prior to Conservatively Staged Excision. JAMA Dermatol. 2018.

19. Mataca E, Migaldi M, Cesinaro AM. Impact of Dermoscopy and Reflectance Confocal Microscopy on the Histopathologic Diagnosis of Lentigo Maligna/Lentigo Maligna Melanoma. Am J Dermatopathol. 2018. 
20. Akay BN, Kocyigit P, Heper AO, Erdem C. Dermatoscopy of flat pigmented facial lesions: diagnostic challenge between pigmented actinic keratosis and lentigo maligna. Br J Dermatol. 2010;163(6):1212-1217. [PubMed: 21083845]

21. de Carvalho N, Farnetani F, Ciardo S, et al. Reflectance confocal microscopy correlates of dermoscopic patterns of facial lesions help to discriminate lentigo maligna from pigmented nonmelanocytic macules. Br J Dermatol. 2015;173(1):128-133. [PubMed: 25413382]

22. Persechino F, De Carvalho N, Ciardo S, et al. Folliculotropism in pigmented facial macules: Differential diagnosis with reflectance confocal microscopy. Exp Dermatol. 2018;27(3):227-232. [PubMed: 29274094]

23. Moscarella E, Rabinovitz H, Zalaudek I, et al. Dermoscopy and reflectance confocal microscopy of pigmented actinic keratoses: a morphological study. J Eur Acad Dermatol Venereol. 2015;29(2):307-314. [PubMed: 24754497]

24. Farrahi F, Egbert BM, Swetter SM. Histologic similarities between lentigo maligna and dysplastic nevus: importance of clinicopathologic distinction. J Cutan Pathol. 2005;32(6):405-412. [PubMed: 15953373]

25. Cohen LM. The starburst giant cell is useful for distinguishing lentigo maligna from photodamaged skin. J Am Acad Dermatol. 1996;35(6):962-968. [PubMed: 8959956]

26. Connolly KL, Giordano C, Dusza S, Busam KJ, Nehal K. Follicular involvement is frequent in lentigo maligna: Implications for treatment. J Am Acad Dermatol. 2019;80(2):532-537. [PubMed: 30266559] 

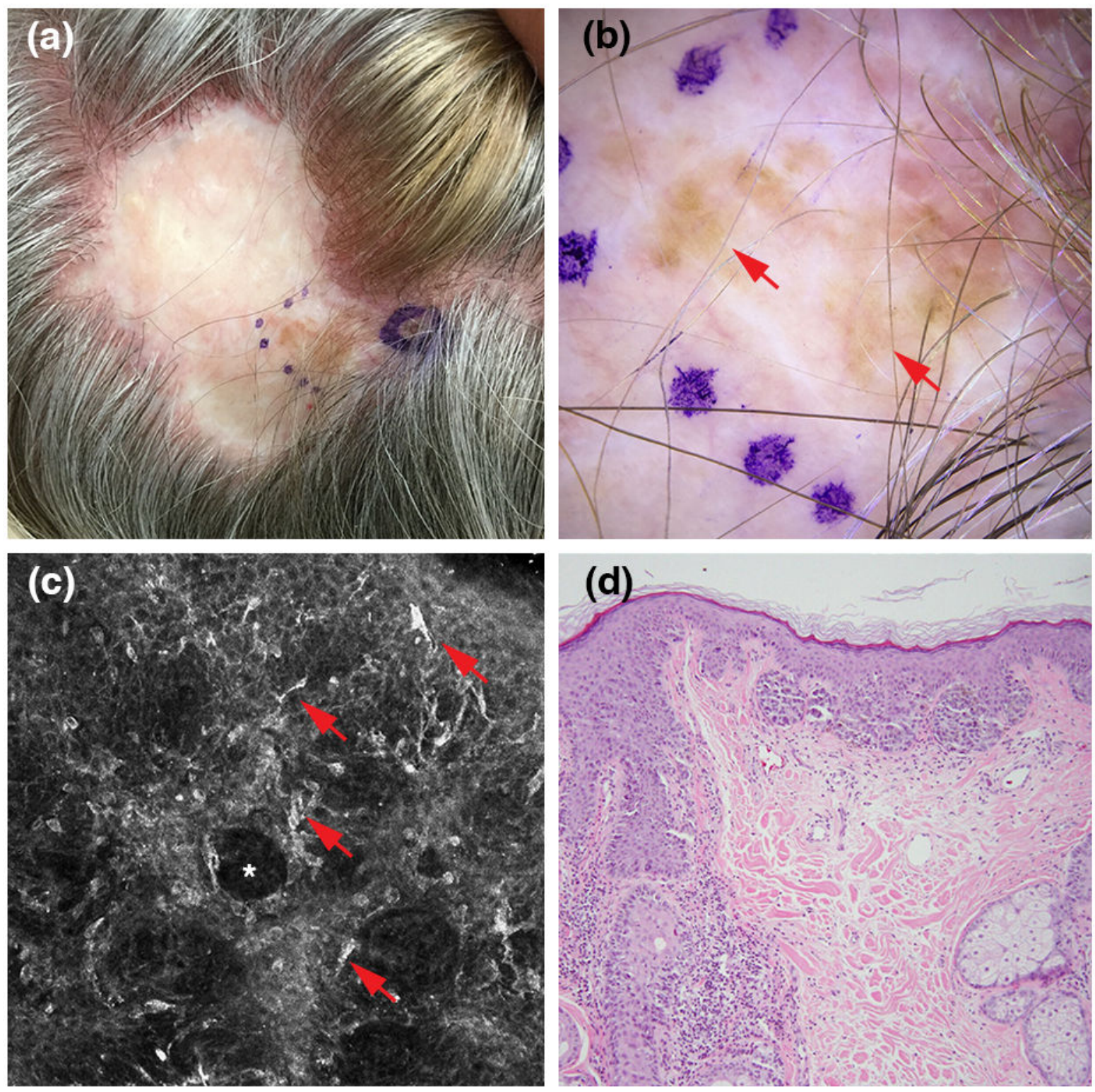

Fig 1.

Recurrent lentigo maligna. (a) Clinical picture showing pigment extends beyond the graft margins. (b) Dermoscopic findings, showing homogeneous or structureless brown areas (red arrows; polarized light dermoscopy; original magnification, 10X). (c) Reflectance confocal microscopy findings, showing round and dendritic bright nucleated cells (red arrows) surrounding hair follicles (white asterisk) $(750 \times 750 \mu \mathrm{m})$. (d) Histopathologically confirmed recurrent lentigo maligna melanoma (Breslow thickness, $0.4 \mathrm{~mm}$; haematoxylin and eosin; magnification, 20X). 

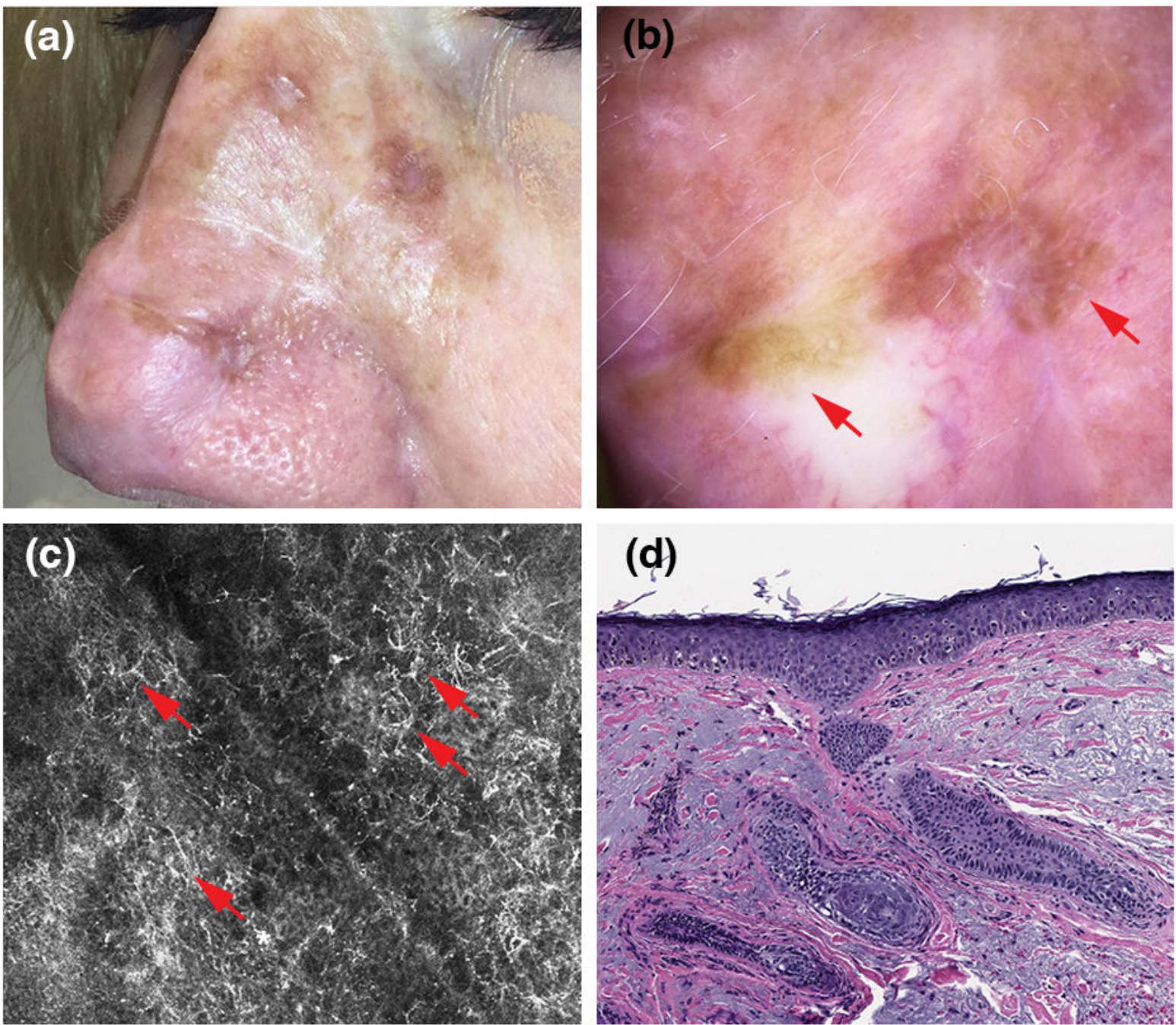

Fig 2.

Recurrent lentigo maligna. (a) Clinical picture showing multiple foci of pigment along the scar of a complex reconstruction site (flap and graft) area on the nose. (b) Dermoscopic findings, showing homogeneous/structureless brown areas (red arrows; polarized light dermoscopy; original magnification, 10X). (c) Reflectance confocal microscopy findings, showing large widespread pagetoid dendritic cells (red arrows; 750 x $750 \mu \mathrm{m}$ ). (d) Histopathologically confirmed recurrent lentigo maligna (red arrow). 

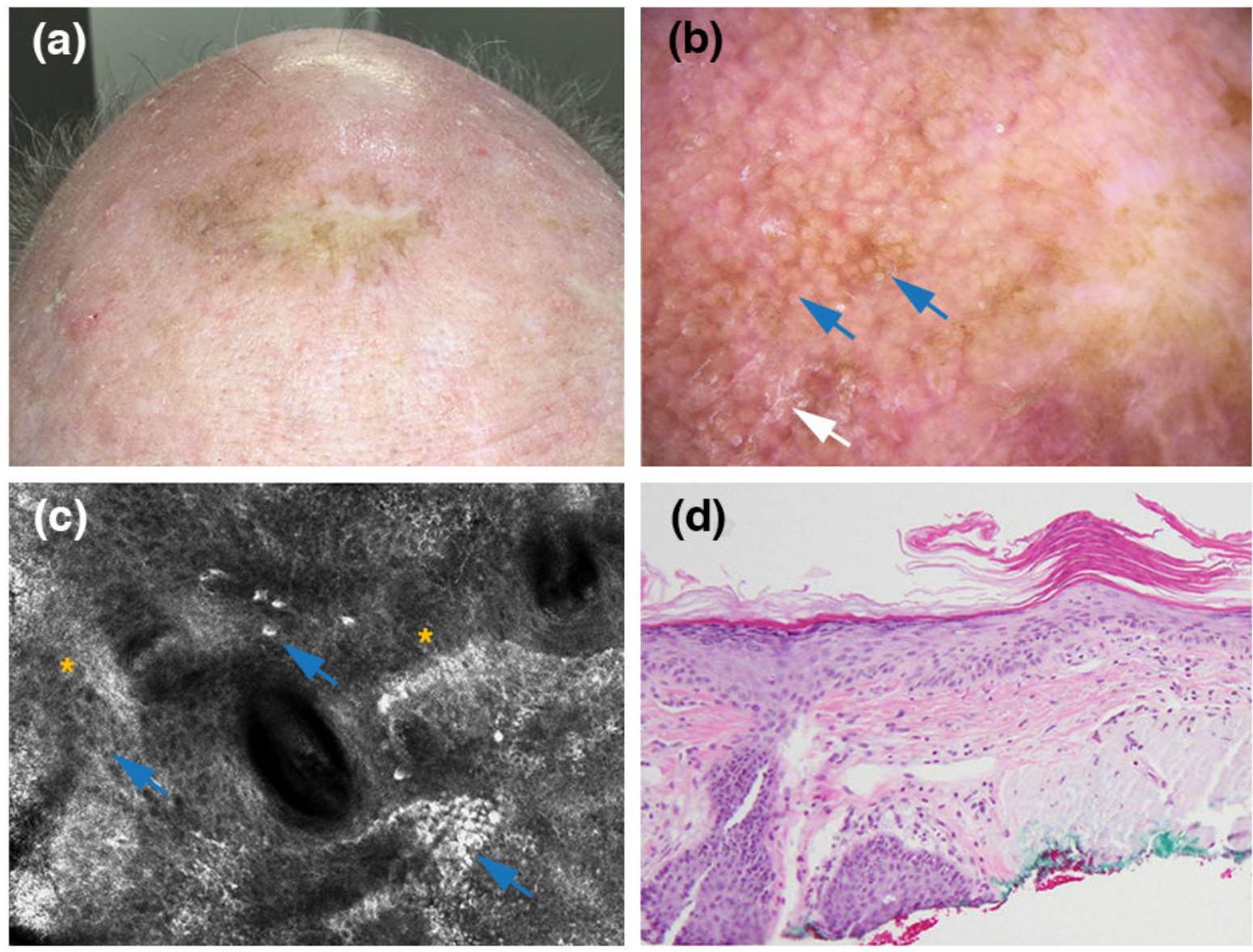

Fig 3.

Pigmented actinic keratosis arising within a lentigo maligna surgical scar. (a) Clinical picture showing pigment extends beyond the graft margins. (b) Dermoscopic findings, showing a pseudonetwork (blue arrows) and scale (white arrow) (polarized light dermoscopy; original magnification, 10X). (c) Reflectance confocal microscopy findings, showing pigmented keratinocytes (blue arrows) and an overall atypical honeycomb pattern (yellow asterisks) $(750 \times 750 \mu \mathrm{m})$. (d) Histopathologically confirmed pigmented actinic keratosis (haematoxylin and eosin; magnification, 20X). 

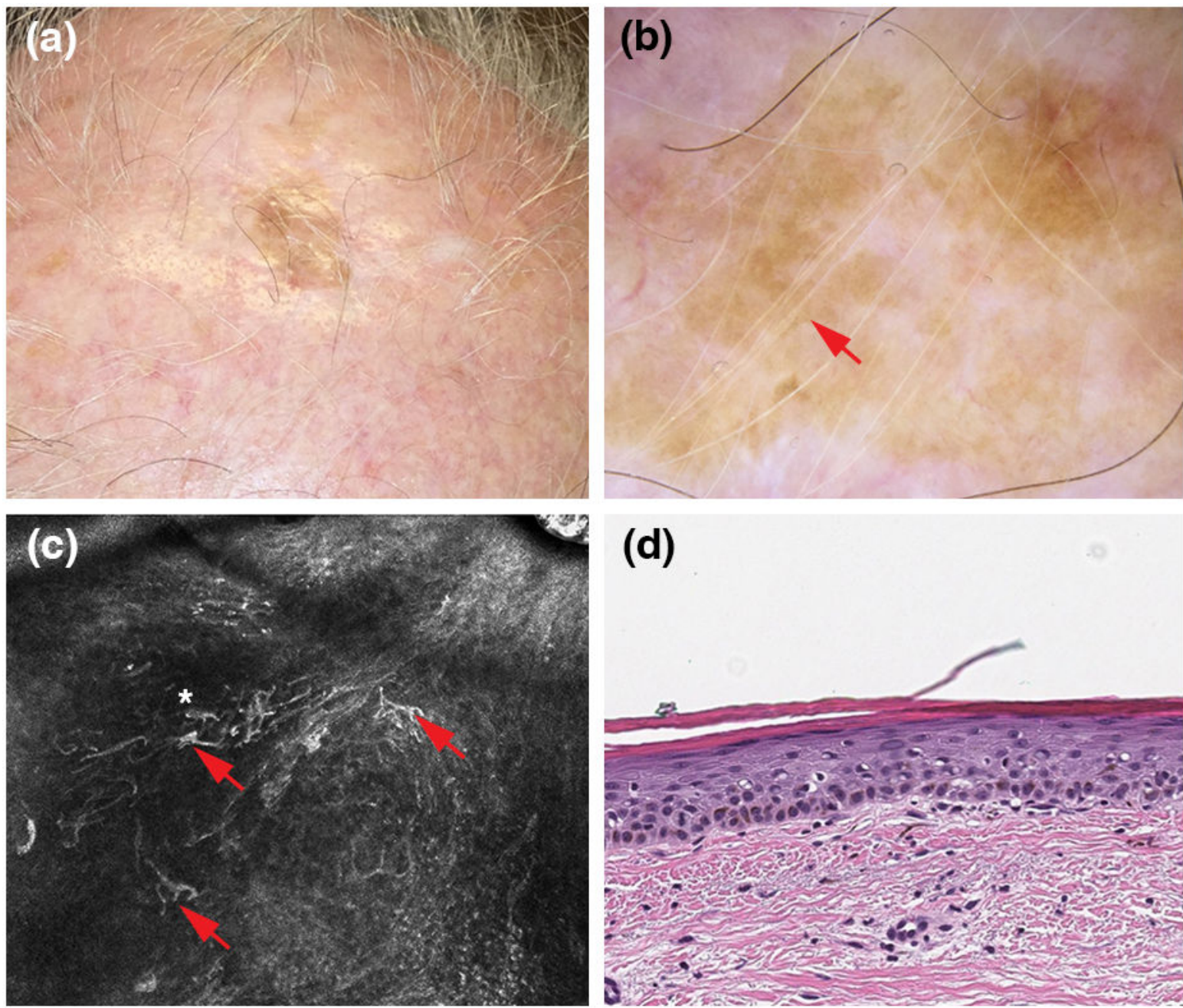

\section{(d)}

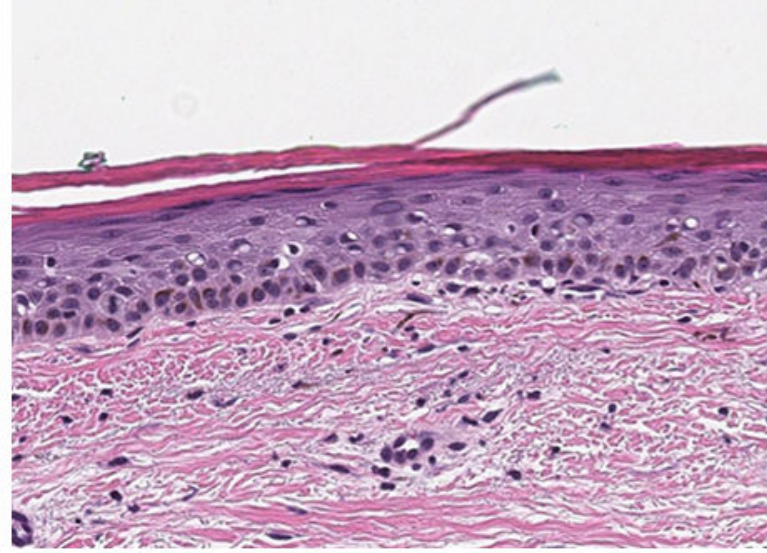

Fig 4.

Pigmented actinic keratosis arising within a lentigo maligna surgical scar. (a) Clinical picture showing pigment extends into the grafted area. (b) Dermoscopic findings, showing a structureless brown pattern (red arrow). No scale is seen. (polarized light dermoscopy; original magnification, 10X) (c) Reflectance confocal microscopy findings, displaying bright large nucleated dendritic cells (red arrows) in a disarranged dermoepidermal junction/ suprabasal epidermis (white asterisk) $(750 \times 750 \mu \mathrm{m})$. (d) Histopathologically confirmed pigmented actinic keratosis (haematoxylin and eosin; magnification, 20X). 


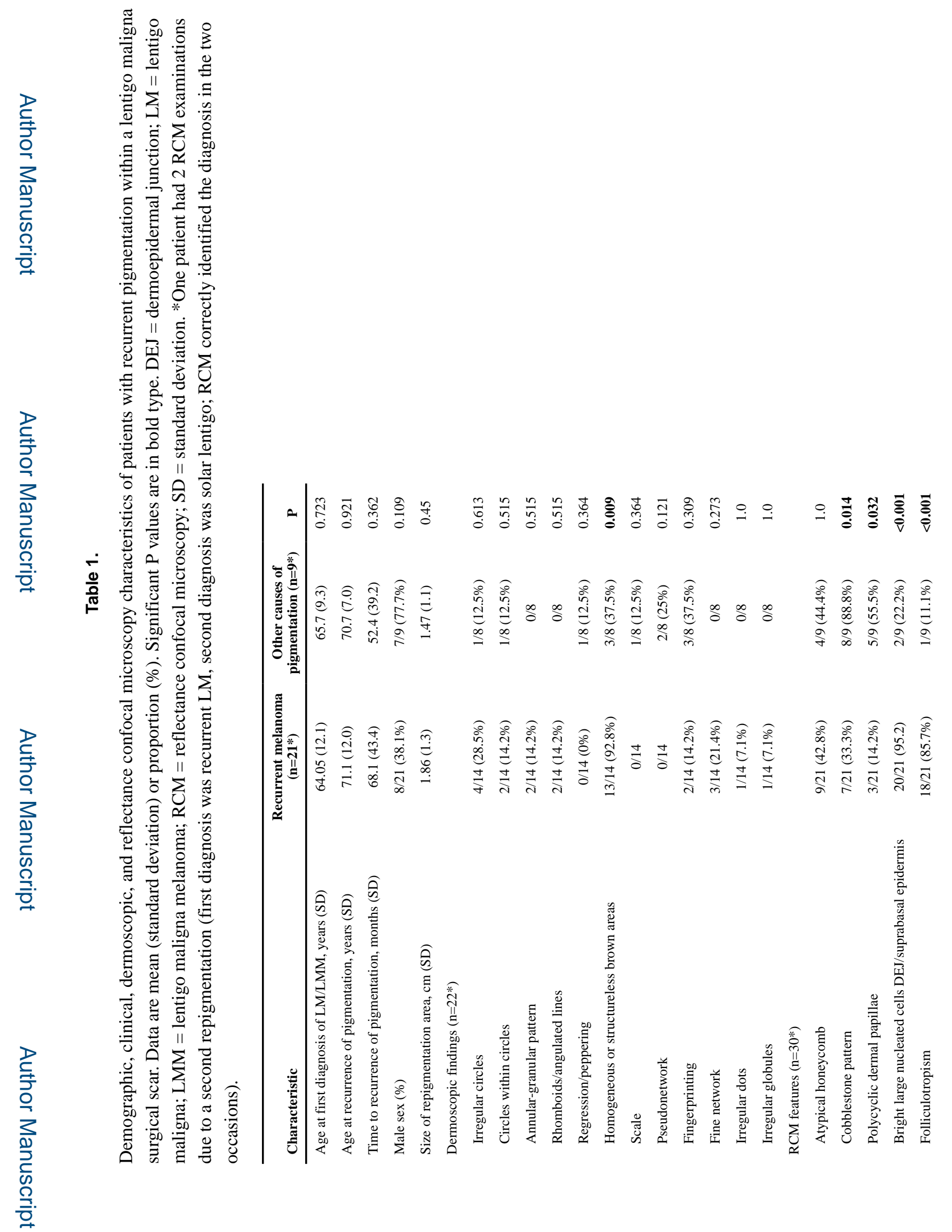

J Eur Acad Dermatol Venereol. Author manuscript; available in PMC 2021 January 01. 

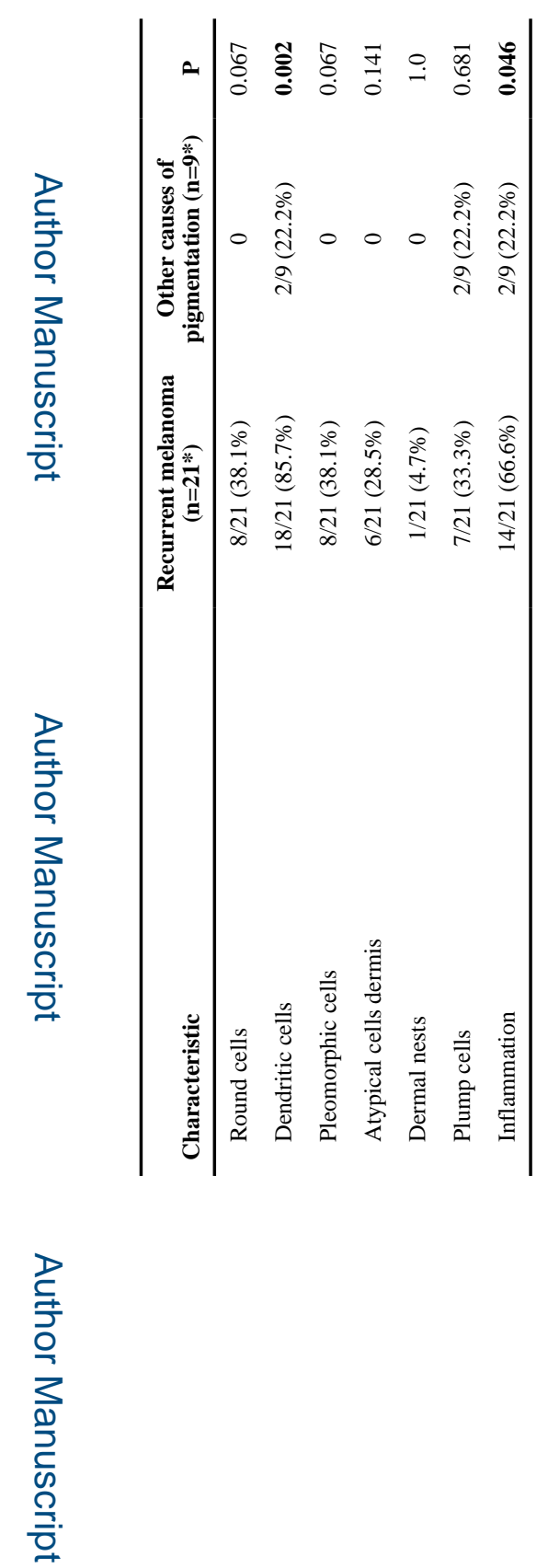

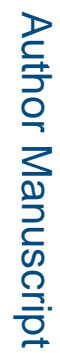

J Eur Acad Dermatol Venereol. Author manuscript; available in PMC 2021 January 01. 